\title{
A threshold-based cloud mask for the high-resolution visible channel of Meteosat Second Generation SEVIRI
}

\author{
S. Bley and H. Deneke \\ Leibniz Institute for Tropospheric Research (TROPOS), Permoserstraße 15, 04318 Leipzig, Germany \\ Correspondence to: S. Bley (bley@tropos.de) \\ Received: 18 January 2013 - Published in Atmos. Meas. Tech. Discuss.: 20 March 2013 \\ Revised: 19 August 2013 - Accepted: 2 September 2013 - Published: 18 October 2013
}

\begin{abstract}
A threshold-based cloud mask for the highresolution visible $(\mathrm{HRV})$ channel $\left(1 \times 1 \mathrm{~km}^{2}\right)$ of the Meteosat SEVIRI (Spinning Enhanced Visible and Infrared Imager) instrument is introduced and evaluated. It is based on operational EUMETSAT cloud mask for the low-resolution channels of SEVIRI $\left(3 \times 3 \mathrm{~km}^{2}\right)$, which is used for the selection of suitable thresholds to ensure consistency with its results. The aim of using the HRV channel is to resolve small-scale cloud structures that cannot be detected by the low-resolution channels. We find that it is of advantage to apply thresholds relative to clear-sky reflectance composites, and to adapt the threshold regionally. Furthermore, the accuracy of the different spectral channels for thresholding and the suitability of the HRV channel are investigated for cloud detection. The case studies show different situations to demonstrate the behavior for various surface and cloud conditions.

Overall, between 4 and $24 \%$ of cloudy low-resolution SEVIRI pixels are found to contain broken clouds in our test data set depending on considered region. Most of these broken pixels are classified as cloudy by EUMETSAT's cloud mask, which will likely result in an overestimate if the mask is used as an estimate of cloud fraction. The HRV cloud mask aims for small-scale convective sub-pixel clouds that are missed by the EUMETSAT cloud mask. The major limit of the HRV cloud mask is the minimum cloud optical thickness (COT) that can be detected. This threshold COT was found to be about 0.8 over ocean and 2 over land and is highly related to the albedo of the underlying surface.
\end{abstract}

\section{Introduction}

The effect of clouds on radiative fluxes depends on cloud type and can vary strongly both in space and time. Accurate information about the physical and radiative properties of clouds is necessary to determine the role of clouds in the climate system including their response to anthropogenic forcings (e.g., Forster et al., 2007).

Geostationary satellite imagers such as Meteosat SEVIRI (Spinning Enhanced Visible and Infrared Imager) are wellsuited to monitor the temporal development of clouds, and to resolve their diurnal cycle fully over land and ocean (Roebeling and van Meijgaard, 2009). The spatial resolution of SEVIRI's narrowband channels $\left(3 \times 3 \mathrm{~km}^{2}\right)$ lags behind that of polar orbiting imagers such as MODIS $(1 \times 1$ down to $\left.0.25 \times 0.25 \mathrm{~km}^{2}\right)$ and AVHRR $\left(1.1 \times 1.1 \mathrm{~km}^{2}\right)$, which limits its ability to resolve small-scale structures. SEVIRI does however have a high-resolution visible (HRV) channel with a nadir resolution of $1 \times 1 \mathrm{~km}^{2}$.

The HRV channel contains important information for studying the small scale variability of clouds and the underlying surface (e.g., Klüser et al., 2008; Deneke and Roebeling, 2010). The study of Derrien et al. (2010a) improves the detection of small-scale low clouds by use of the HRV channel. The HRV channel reflectance was also used by Carbajal Henken et al. (2011) for the detection of deep convective clouds. Nevertheless, few operational products based on the HRV channel are available, which is a significant hurdle for use of its finer spatial resolution for scientific studies and applications.

The estimation of cloud and/or surface properties from multispectral satellite images requires the classification of pixels into cloud-free and cloudy classes as an initial step. 
Most cloud detection algorithms described in the literature rely on a combination of threshold tests applied to different spectral channels for this purpose. Rossow et al. (1989) present an overview of early methods chosen for cloud masking. These methods often exploit the fact that clouds generally appear brighter in solar channels due to reflection, and colder in infrared channels relative to cloud-free surfaces. In addition, spatial coherence tests are commonly used, as clouds are often more variable than the underlying surface (e.g., see Saunders and Kriebel, 1988). It should be noted that spatial coherence tests also rely on thresholds for identifying regions with high variability.

Cloud masking is one particular case of object identification by thresholding, and can be described mathematically by considering the grey-level histograms based on specific channel radiances. Thresholds are selected to separate the histograms for the cloud-free and cloudy pixels of a satellite image best. Suitable thresholds for satellite channels are often selected by experts (e.g., Saunders and Kriebel, 1988). As an alternative, automatic statistical methods can be applied to select optimal thresholds. Here, methods that make use of a training data set for threshold selection (supervised methods) need to be differentiated from those that select thresholds based on intrinsic properties of the data set (unsupervised methods). Yang et al. (2007) have investigated several algorithms for unsupervised threshold selection, and have determined the most accurate ones for application to cloud masking for the multi-angle imaging spectroradiometer (MISR) over land. Regardless of the method, independent reference data are needed to establish the accuracy of threshold-based classification methods.

The goal of the present paper is to develop a cloud mask based on the HRV channel, which exploits its high spatial resolution, and is suitable to study small-scale features of clouds, including, for example, their horizontal dimensions. This does preclude the use of spatial coherence tests due to their non-local nature. Instead, a differencing approach using clear-sky composite reflectances as a background is adopted to improve the contrast between clear-sky and cloudy situations (e.g., Minnis and Harrison, 1984; Ipe et al., 2003). Nevertheless, a threshold test applied to a single visible channel cannot achieve the accuracy of other SEVIRI cloud masks that are based on multiple spectral channels. Instead of replicating other cloud mask algorithms, this HRV mask is designed as a complement to an existing cloud mask used as a reference for threshold selection and to estimate the mask's accuracy. The operational cloud mask product (CLM) by EUMETSAT's Meteorological Product Extraction Facility, which is based on the narrowband channels of SEVIRI (EUMETSAT, 2007), is a convenient choice for this purpose as it is distributed through the EUMETCast system together with the level 1.5 SEVIRI images, and it is used in our study.

This paper is structured as follows: in Sect. 2, a brief overview of the data sets used in our study, including the characteristics of the SEVIRI instrument, is given. This is followed by Sect. 3, which describes our proposed cloud masking method. Section 4 presents results and discussions, followed by conclusions and an outlook in Sect. 5 .

\section{Data}

\subsection{Instrumental data}

The current series of European geostationary satellites, Meteosat Second Generation (MSG), is operated by EUMETSAT. Its main payload is the the Spinning Enhanced Visible and Infrared Imager (SEVIRI), an optical imaging radiometer. Three MSG satellites, Meteosat-8 to Meteosat-10, were launched and are positioned in geostationary orbit at an altitude of $36000 \mathrm{~km}$ above the Equator. Meteosat-9 observes the full disk of the earth as a primary geostationary service with a repeat cycle of $15 \mathrm{~min}$. Meteosat- 8 is currently used as a standby and operates the rapid-scan service covering Europe with a 5 min repeat cycle. Meteosat- 10 was launched on 5 July 2012, and is currently in commissioning. A detailed description of MSG is given by Schmetz et al. (2002).

The SEVIRI instrument has 3 solar channels $(0.6,0.8$ and $1.6 \mu \mathrm{m}), 8$ thermal infrared channels $(3.9,6.2,7.3,8.7,9.7$, $10.8,12.0$ and $13.4 \mu \mathrm{m})$ and one high-resolution visible channel (HRV) $(0.4-1.1 \mu \mathrm{m})$. The nadir spatial resolution for SEVIRI's HRV channel is $1 \times 1 \mathrm{~km}^{2}$ and $3 \times 3 \mathrm{~km}^{2}$ for the other 11 channels. However, the true optical resolution is lower by a factor of about 1.6 for both high resolution and lowresolution (LRES) channels (Schmetz et al., 2002).

Only the $0.6,0.8,1.6,8.7 \mu \mathrm{m}$ and HRV channels are considered in this study. The normalized spectral response functions of the narrowband solar and the broadband HRV channels are shown in Fig. 1.

The narrowband channels cover the full disk of the earth with $3712 \times 3712$ pixels. At a 3 -fold higher resolution, this results in a nominal image size of $11136 \times 11136$ pixels for the HRV channel. However, the actual HRV channel coverage is only 5568 pixels in east-west direction. An upper region of 3072 scanlines with a fixed position is centered on Europe. The lower region consisting of 8064 scan lines follows the daily illumination. Only the upper region is considered in this study.

\subsection{EUMETSAT cloud mask}

The EUMETSAT cloud mask (CLM) is derived by the Meteorological Product Extraction Facility (MPEF) and utilizes a combination of several multi-spectral threshold tests grouped into different categories to distinguish between cloudy and cloud-free pixels (see EUMETSAT, 2007, for a detailed description of the algorithm). The CLM is an operational SEVIRI product and is derived every $15 \mathrm{~min}$ for the full disk. In the final product, each pixel is labeled either as 0 (clear-sky ocean), 1 (clear-sky land), 2 (cloudy) or 3 (no data). The EUMETSAT threshold tests involve almost every 


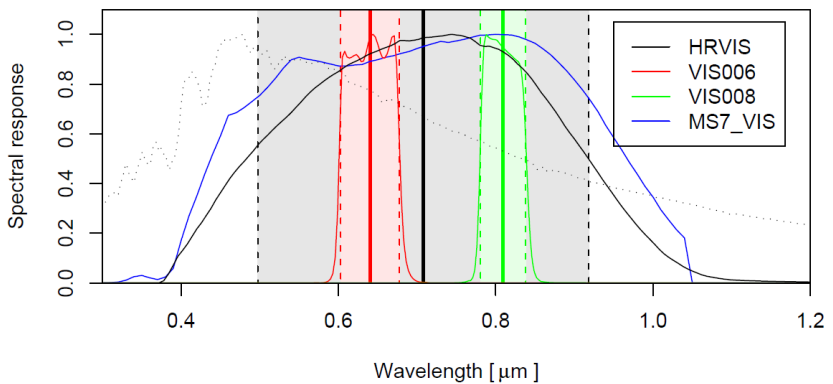

Fig. 1. Normalized spectral response functions of the Meteosat-9 SEVIRI radiometer for the $0.6 \mu \mathrm{m}$ (red), $0.8 \mu \mathrm{m}$ (green) and HRV (black) channels. The central wavelength of each channel is marked by a thick colored line, and the spectral region covered by the channel width has been shaded. The solar spectrum is added as a dotted line.

SEVIRI channel with the exception of channel $8(9.7 \mu \mathrm{m})$, as it is mainly sensitive to tropospheric and stratospheric ozone and thus adds little additional information for cloud masking, and channel 12 (HRV), as it is not available for the full disk.

\subsection{Study regions}

To evaluate the threshold-based cloud mask algorithm and study its performance for different surface types and synoptic conditions, we have selected the following four regions in and around Europe: (1) Atlantic, (2) the Alps, (3) Upper Rhine Valley and (4) Spain (see Fig. 2). The four regions comprise $192 \times 192 \mathrm{HRV}$ pixels (or $64 \times 64$ LRES pixels) to provide enough data points to calculate representative histogram functions. This size has been chosen as a reasonable trade-off versus the advantage offered by a smaller size and thus a smaller surface variability. To illustrate the improvements gained by applying thresholds relative to a clear-sky composite, we have focused on regions with a relatively high spatial surface variability such as the Alps and the Upper Rhine Valley. Due to our future plans to investigate the diurnal cycle of shallow cumulus convection we use the Rapid Scan Service (RSS) of Meteosat over the European region. The presented algorithm is limited in terms of the surface albedo, which is discussed in Sect. 3 (Fig. 4).

\section{Methods and algorithms}

For applying a binary classification to separate cloudy and clear-sky pixels, we rely on the following simplified assumption. In general clouds have a higher HRV reflectance compared to the clear-sky surface and thus appear brighter. Counterexamples include snow-covered surfaces, enhanced clearsky reflectances due to aerosols, and cloud shadows, but they are neglected here. A perfect classification would allow an exact separation between clear-sky and cloudy reflectances based on a single reflectance threshold.

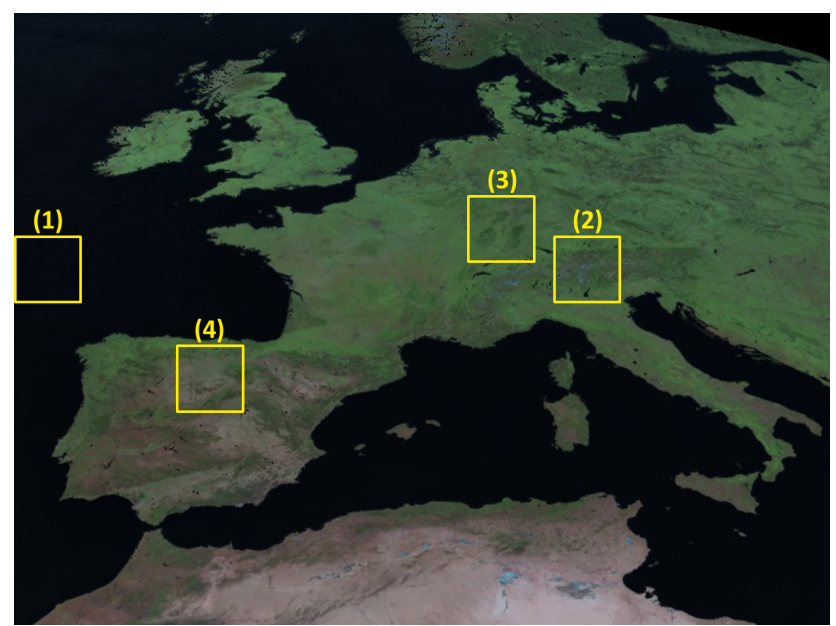

Fig. 2. Day natural color (RGB) clear-sky composite of Lensky and Rosenfeld (2008) based on clear-sky reflectances of the 0.6, 0.8 and $1.6 \mu \mathrm{m}$ channel. Median values of clear-sky reflectance over a period of 16 days (1-16 June 2011) and cloud-cleared with the EUMETSAT cloud mask are shown (at standard SEVIRI resolution). The following four regions used in our study are marked by boxes: (1) Atlantic, (2) the Alps, (3) Upper Rhine Valley and (4) Spain.

The first step in this study is the separation of clear-sky and cloudy HRV pixels based on the EUMETSAT cloud mask. The latter includes multiple solar and thermal threshold tests. As it is not based on a fixed reflectance threshold, clear-sky pixels can have a higher HRV reflectance than cloudy pixels. Consequently, there is an overlap between the histograms of the clear-sky and the cloudy reflectances (Fig. 3). The normalized frequency distribution of clear-sky and cloudy HRV reflectances are shown for the regions Spain (yellow), Upper Rhine Valley (green), the Alps (red) and Atlantic (blue). The clear-sky histograms have been calculated as a median value over a 16-day period (1-16 June 2011). Figure 3 shows the lowest clear-sky reflectance and lowest variability over the Atlantic region. The clear-sky reflectance histogram over Spain reveals a high value and simultaneously a high spatial variability. Readers should note the overlap between the clear and cloudy histograms.

Several reasons can cause the broadness of the clear-sky histogram and thus the overlap in the HRV reflectance histograms (Fig. 3). This includes spatial and temporal variability of the surface reflectance (e.g., due to changes in vegetation, atmospheric aerosols). Additionally undetected thin cirrus clouds with low visible reflectance can contaminate the HRV clear-sky histogram. This overlap is the major source of uncertainty for our cloud mask. The challenge is thus to reduce this overlap, and to find an optimal threshold to obtain the best classification.

One of the major problems that causes the overlap is the broadness of the cloudy histogram due to different cloud types with different COTs. This broadness is strongly related 


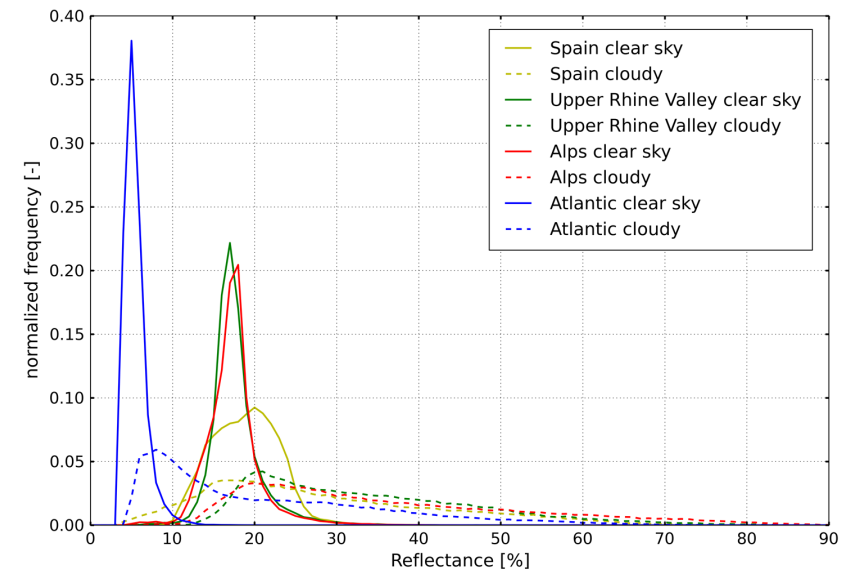

Fig. 3. Normalized frequency distribution of cloudy and clear-sky HRV reflectances over Spain (yellow), Upper Rhine Valley (green), the Alps (red) and Atlantic (blue) observed by MSG SEVIRI. Separation between cloudy (dotted line) and clear-sky (solid line) reflectances is based on EUMETSAT cloud mask. EUMETSAT cloud mask is upsampled to HRV resolution.

to the definition of the cloud the user wants to capture with his/her cloud mask. In our study it is related to the minimum COT that can be detected reliably by the HRV cloud mask depending on the underlying surface albedo. In order to quantify the accuracy of the cloud detection algorithm and the implication of the underlying surface, we calculate the minimum COT using a simple, qualitative model (Eq. 1 by Lacis and Hansen, 1974). The change in the planetary albedo due to the occurrence of clouds is based on an expression by Liou (1980) to take into account multiple scattering between the cloud and the underlying surface. Furthermore, no absorption within the cloud is considered in this formula:

$A_{\mathrm{P}}=A_{\mathrm{C}}+R_{\mathrm{S}} \cdot \frac{\left(1-A_{\mathrm{C}}\right)^{2}}{1-R_{\mathrm{S}} \cdot A_{\mathrm{C}}}$.

Thereby $A_{\mathrm{P}}$ is the change in the planetary albedo, $A_{\mathrm{C}}$ the cloud albedo and $R_{\mathrm{S}}$ the albedo of the underlying surface. The cloud albedo can be approximated by

$A_{\mathrm{C}}=\frac{\tau_{\mathrm{c}}}{\tau_{\mathrm{c}}+7.7}$

where $\tau_{\mathrm{c}}$ is the cloud optical thickness (Lacis and Hansen, 1974). The statement of Eq. (1) is illustrated by Fig. 4 for four different COTs. The curves show the change in planetary albedo due to the occurrence of clouds with a particular COT from 0.2 to 2 over a surface with different clear-sky reflectances between 0 and 0.55 . $\delta$ (planetary albedo - surface albedo) indicates the accuracy of the threshold that is necessary to detect a cloud over a specific ground. The thin blue area $(\mathrm{COT}=0.2-0.5)$ illustrates that a high accuracy of the threshold is necessary to detect those optical thin clouds. A smaller $\delta$ (planetary albedo - surface albedo) is related to a

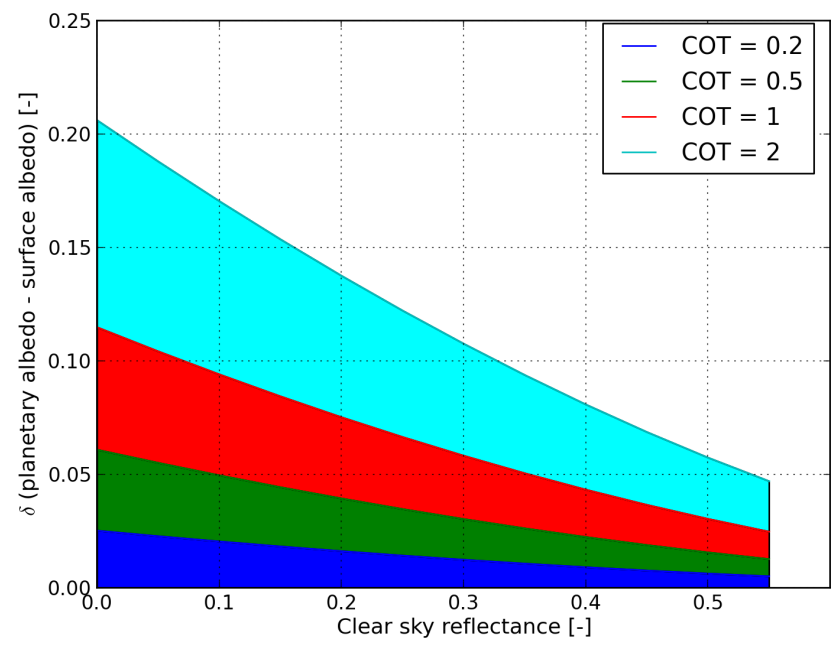

Fig. 4. Change in planetary albedo due to the occurrence of clouds with a COT of 0.2 (blue), 0.5 (green), 1 (red) and 2 (cyan) as a function of the clear-sky reflectance of the underlying surface. The $\delta$ ( $y$ axis) demonstrates the accuracy that is necessary to detect a cloud over a surface with a specific clear-sky reflectance. This simple model is based on Eq. (1) by Lacis and Hansen (1974).

higher accuracy. This accuracy has to be even higher with an increasing clear-sky reflectance of the underlying surface (Fig. 4). For a higher COT the $\delta$ (planetary albedo - surface albedo) is obviously higher, although substantially decreasing with a decreasing clear-sky reflectance.

Applying Eq. (2) to the average clear-sky reflectances (Table 2) for our four regions results in a minimum ascertainable COT of $0.88,2.62,2.62$ and 2.95 over the Atlantic, the Alps, the Upper Rhine Valley and Spain during the summer period. This result demonstrates a negligence of thin cirrus clouds over land solely with the solar channel information. In summary, it can be stated that for a clear-sky reflectance higher than 0.25 it is very difficult to detect clouds above such bright surfaces. Over surfaces with about 0.35 clear-sky reflectances or even higher, no accurate cloud mask can be derived (Fig. 4). Equation (2), Fig. 4 and Table 2 indicate that no cloud with a COT above 1 can be detected reliably by the HRV cloud mask over the regions that are investigated in this study.

\subsection{Cloud-free composites}

To reduce the uncertainties caused by spatially varying surface reflectance, we apply the thresholds for cloud detection relative to a composite map of clear-sky HRV reflectance. These maps are derived initially based on the EUMETSAT cloud mask. Due to the lower SEVIRI standard resolution, it is necessary to upsample the EUMETSAT cloud mask to the 3 times higher resolution of the HRV channel. This is done using nearest-neighbor interpolation. The clear-sky composite is based on the median value $\tilde{r}_{\mathrm{cs}}$ of all clear-sky 
Table 1. Contingency table with binary classification cloudy and clear.

\begin{tabular}{lll}
\hline & $\begin{array}{ll}\text { EUMETSAT } \\
\text { CLM cloudy }\end{array}$ & $\begin{array}{l}\text { EUMETSAT } \\
\text { CLM clear }\end{array}$ \\
\hline $\begin{array}{l}\text { Predicted HRV cloudy } \\
\text { Predicted HRV clear }\end{array}$ & $\begin{array}{ll}\text { true positive } \\
\text { false negative }\end{array}$ & $\begin{array}{l}\text { false positive } \\
\text { true negative }\end{array}$ \\
\hline
\end{tabular}

reflectances observed during a 16-day period. The median is chosen as an alternative to the average value to neglect subpixel small-scale clouds, which are labeled as clear by the MPEF cloud mask. The average value would include biases such as small-scale undetected clouds or cloud shading. The length of this period seems appropriate to ensure relatively constant surface conditions and a high likelihood of finding at least one cloud-free observation for each pixel. Nevertheless, pixels can occur with no clear-sky observation due to persistent clouds. Such HRV pixels are always reported as cloudy.

Instead of subtracting the clear-sky composite from the observed reflectance, an anomaly map is created for each $192 \times 192$ pixel region using Eq. (3):

$r_{\text {norm }}(x, t)=r_{\mathrm{obs}}(x, t)-\left(\left\langle\tilde{r}_{\mathrm{cs}}(x)\right\rangle_{t}-\left\langle\tilde{r}_{\mathrm{cs}}\right\rangle_{t, x}\right)$.

Here, $r_{\mathrm{obs}}(x, t)$ is the observed HRV reflectance field at time $t$ during a specific period. The subtrahend $\left\langle\tilde{r}_{\mathrm{cs}}(x)\right\rangle_{t}-\left\langle\tilde{r}_{\mathrm{cs}}\right\rangle_{t, x}$ consists of two parts. The first term $\left\langle\tilde{r}_{\mathrm{cs}}(x)\right\rangle_{t}$ is the spatially resolved temporal median of the clear-sky reflectance, while the second term $\left\langle\tilde{r}_{\mathrm{cs}}\right\rangle_{t, x}$ is the spatial and temporal median of the clear-sky reflectance.

Figure 5 demonstrates the effects of reducing the spatial variability by applying the clear-sky composite anomaly map. The solid line shows the histogram of the clear-sky HRV reflectance over Spain observed by MSG SEVIRI on 15 July 2011 and derived by applying the EUMETSAT cloud mask. The dotted line represents the normalized clear-sky histogram.

The effect of this treatment is illustrated by the green arrows in Fig. 5. The distribution indeed becomes more narrow, which indicates that our method is capable of compensating for the spatial variability of the underlying surface reflectance. This method therefore minimizes the overlap between the cloudy and clear histograms and reduces the associated uncertainty of the HRV cloud mask.

\subsection{Optimal threshold}

The threshold for detecting cloudy pixels should maximize the quality of our classification. It is thus necessary to compare it to reference data, and to define suitable quality criteria for assessing its accuracy. The four possible outcomes for comparing two binary classifications are listed in the contingency Table 1 . In this study the EUMETSAT cloud mask is used as a reference, and the predicted class of the HRV

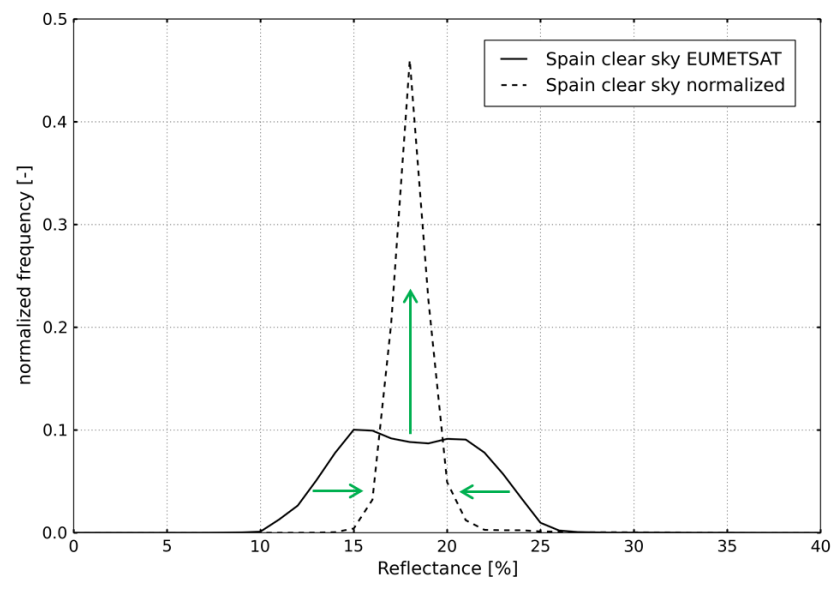

Fig. 5. Normalized frequency distribution of clear-sky HRV reflectances over Spain observed by MSG SEVIRI on 15 July 2011. The solid line is the distribution of clear-sky reflectances identified by the EUMETSAT cloud mask. The dotted line is the histogram of the normalized clear-sky reflectance after subtraction of the clearsky composite anomaly map. The green arrows symbolize the reduction of spatial variability.

cloud mask depends on the selected reflectance threshold. Due to the use of prior information, this method corresponds to a supervised classification algorithm. For determining the optimal threshold, a suitable measure is sought that combines the frequencies of the four outcomes into one scalar quantity.

One measure that meets our requirements is the Matthews correlation coefficient (MCC, Matthews, 1975). Like the Pearson correlation coefficient for the continuous case, it quantifies the correlation between two binary variables in a range from -1 to 1 , with 1 corresponding to perfect agreement.

The MCC is defined as follows:

$\mathrm{MCC}=\frac{\mathrm{tp} \cdot \mathrm{tn}-\mathrm{fp} \cdot \mathrm{fn}}{\sqrt{(\mathrm{tp}+\mathrm{fp}) \cdot(\mathrm{tp}+\mathrm{fn}) \cdot(\mathrm{tn}+\mathrm{fp}) \cdot(\mathrm{tn}+\mathrm{fn})}}$.

It can be calculated directly from the contingency table from Eq. (4). One advantage of the MCC is its insensitivity to the frequency of both classes. This ensures that our cloud mask performs well in regions and seasons with low, medium and high frequency of clouds. The threshold that corresponds to the maximum of the MCC is chosen as optimal.

The flow chart in Fig. 6 visualizes the HRV cloud mask algorithm. The first step is the calculation of the clear-sky composite. This clear-sky composite consists of HRV reflectances, but the assignment between clear-sky and cloudy pixels is based on the MPEF cloud mask (see Sect. 3.1 for details). In the next iteration we consider the sub-pixel cloud coverage in the MPEF cloud mask to get a clear-sky composite that is based on detected clear-sky HRV pixels. The normalized HRV reflectance field improves with a higher accuracy of the clear-sky composite (Fig. 6, middle plot on the right panel). The variability of the cloudy histogram is 


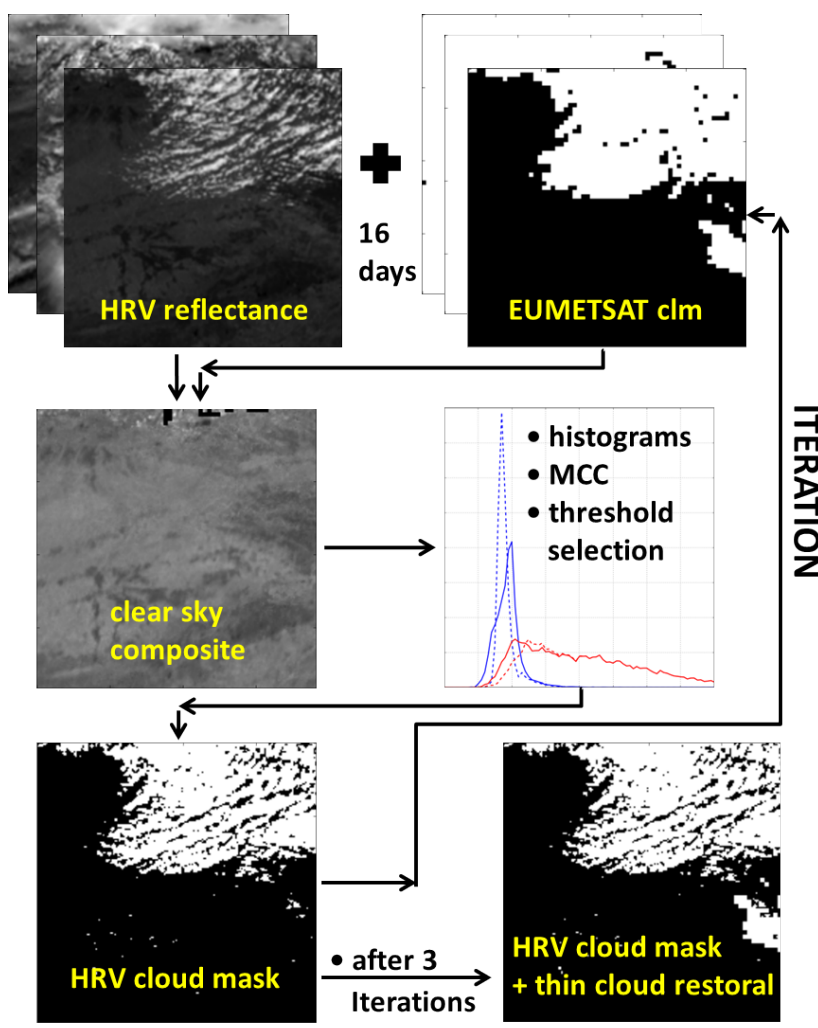

Fig. 6. Flow chart of our HRV cloud mask algorithm, based on the HRV reflectance and the EUMETSAT cloud mask as inputs. The clear-sky composite is initially calculated based on the HRV reflectance and the EUMETSAT cloud mask for 16-day periods. The panel showing the histograms represents the threshold selection step based on maximizing the MCC. As a final step, the thin cloud restoral to consider thin clouds is carried out. An iterative approach including the HRV cloud mask is chosen for the calculation of the clear-sky composite for consistency.

decreased as well, because thin cirrus clouds below the defined minimum COT are no longer considered by the HRV cloud mask (Fig. 6).

The calculation of the quality criteria is based on the MPEF cloud mask and the normalized HRV reflectance field. The corresponding reflectance of the maximum of the MCC is defined as the relative threshold $t_{\text {rel }}$; $t_{\text {rel }}$ of each iteration is then applied to the reflectance field and results in a higher accuracy of the HRV cloud detection algorithm. The MPEF cloud mask is the constant reference mask for this calculation. The resulting HRV cloud mask of this algorithm is used as new input for the whole procedure. The iteration algorithm will stop if the deviation of the relative threshold between two iterations is below 0.001 .

A final processing step is introduced to consider thin clouds with a thin cloud restoral. The detection of thin cirrus clouds solely based on the broadband information from the HRV channel is difficult. As it is our aim to determine a HRV cloud mask that is complementary to and consistent with the MPEF cloud mask, we redefine $3 \times 3$ clear-sky HRV pixel blocks as cloudy if the appropriate pixel of the MPEF cloud mask detects clouds. These redefined HRV pixels are flagged in a different way than cloudy pixels that are introduced as cloudy from the HRV cloud detection algorithm (Fig. 6). This is mainly done due to the fact that this gives the user of the cloud mask the choice of what cloud type will be investigated depending on the application (e.g., clear-sky composites). The thin cloud restoral has no impact on smallscale clouds, which are undetected by the MPEF cloud mask. Our future plan is to use the cloud type description of the Support to Nowcasting and Very Short Range Forecasting (NWCSAF) for the investigation of thin cirrus clouds (see Sect. 5 for details).

\section{Results and discussion}

In order to assess the quality of the HRV cloud mask, some aspects related to its accuracy are investigated and discussed in this section. The EUMETSAT cloud mask is used as a reference to support the consistency of both cloud masks, and due to the lack of other suitable reference data. For this analysis, the four regions shown in Fig. 2 are used as typical examples for different surface types.

To compare our results with the EUMETSAT cloud mask, the latter mask has been upscaled to HRV resolution.

First, the cloud detection frequency for pixels in the EUMETSAT cloud mask has been determined as a function of the number of cloudy pixels identified by the HRV cloud mask algorithm in the corresponding $3 \times 3 \mathrm{HRV}$ pixel blocks. Results have been aggregated for each region over the time period from 1 July until 16 August 2011. The result of this comparison is plotted in Fig. 7. For completely cloudy HRV pixel blocks, we find $100 \%$ agreement with the corresponding EUMETSAT cloud mask classification. In contrast, $10 \%$ of all completely clear HRV pixel blocks are actually identified as cloudy by the EUMETSAT cloud mask. Closer inspection of several corresponding scenes revealed that this deviation is mainly caused by optically thin cirrus clouds, which are not detected by the HRV channel due to their low reflectance. These cases are addressed by the thin cloud restoral and motivated its inclusion in the algorithm. Although the thin cloud restoral works well in general, some artifacts can occur under specific circumstances as described below.

One particular case over the Upper Rhine Valley has been chosen to illustrate the complementary information of the MPEF and the HRV cloud mask (Fig. 8). This case example demonstrates very well how both cloud masks gain different information about the cloud types. Misclassified cloud pixels by the HRV cloud mask against detected cloud pixels by the MPEF cloud mask can be explained by optical thin clouds with a COT underneath a critical threshold (Fig. 8, red pixels). Areas that are contaminated with small-scale shallow 
Table 2. Results of the HRV cloud mask algorithm averaged over three 16-day periods starting 1 June, 1 July and 1 August 2011 . The four regions considered are listed in column $1 . \overline{\mathrm{Cc}}$ is the average cloud cover, and $\overline{r_{\mathrm{cs}}}$ is the spatially averaged temporal median HRV clear-sky reflectance including its standard deviation $\operatorname{std}\left(r_{\mathrm{cs}}\right)$. Columns 4 and 5 report the cloud detection thresholds above which a pixel is classified as cloudy. $t_{\text {abs }}$ lists the absolute threshold without use of the HRV clear-sky reflectance composite, while $t_{\text {rel }}$ is the threshold relative to the composite. The percentage deviations between the HRV and the EUMETSAT cloud mask are given in columns 6-8. Here, Dev $v_{\text {abs }}$ and Dev ${ }_{\text {rel }}$

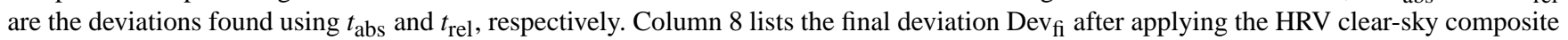
and thin cloud restoral.

\begin{tabular}{lclllrrc}
\hline Regions & $\overline{\mathrm{Cc}}[\%]$ & $\overline{r_{\mathrm{cs}}} \pm \operatorname{std}\left(r_{\mathrm{cs}}\right)[-]$ & $t_{\mathrm{abs}}[-]$ & $t_{\mathrm{rel}}[-]$ & $\operatorname{Dev}_{\mathrm{abs}}$ & $\operatorname{Dev}_{\mathrm{rel}}$ & $\operatorname{Dev}_{\mathrm{fi}}$ \\
\hline Atlantic & 85 & $0.068 \pm 0.007$ & +0.042 & +0.029 & 7.1 & 6.1 & 2.9 \\
The Alps & 75 & $0.187 \pm 0.022$ & +0.080 & +0.051 & 13.6 & 10.1 & 8.4 \\
Upper Rhine Val. & 79 & $0.191 \pm 0.017$ & +0.067 & +0.048 & 10.7 & 8.8 & 6.5 \\
Spain & 40 & $0.193 \pm 0.03$ & +0.085 & +0.044 & 12.4 & 10.3 & 5.3 \\
\hline
\end{tabular}

convection with an optical thickness above our threshold cannot be detected by the MPEF cloud mask (Fig. 8, blue pixels).

The HRV reflectance misses a high amount of cloudy pixels corresponding to thin cirrus clouds. This example demonstrates that the capabilities of the HRV channel for detecting thin cirrus clouds are limited. When considering the brightness temperature difference BT $10.8 \mu \mathrm{m}-\mathrm{BT} 3.9 \mu \mathrm{m}$, the thin cirrus clouds can clearly be recognized in the northwestern corner of the region. Usually the brightness temperature difference BT $10.8 \mu \mathrm{m}-\mathrm{BT} 12.0 \mu \mathrm{m}$ is applied over all surfaces to detect thin cirrus clouds (Derrien et al., 2010b). To give a better feeling of our statement about Fig. 8c, we use the brightness temperature difference BT $10.8 \mu \mathrm{m}-\mathrm{BT} 3.9 \mu \mathrm{m}$ for that, because it shows a better occurrence of thin cirrus clouds in the northwestern corner in our case example (Frey et al., 2008).

The thin cloud restoral redefines a $3 \times 3$ pixel block only as cloudy if the entire block is detected as clear by the threshold algorithm. This approach is problematic for situations where small-scale low-level clouds occur underneath a larger cirrus cloud. This effect is visible in Fig. 8. The EUMETSAT cloud mask and the brightness temperature difference BT $10.8 \mu \mathrm{m}-$ BT $3.9 \mu \mathrm{m}$ indicate a larger cloud coverage due to cirrus. Some brighter pixels appear in the northern HRV image section, which are likely caused by small convective clouds. In the vicinity of these clouds, unrealistic gaps in cloud coverage occur.

The most valuable benefit of the HRV cloud mask is the high proportion of small-scale cumulus clouds that are likely missed by the MPEF cloud mask. This effect is indicated by the blue areas in Fig. 8d. Concerning the frequency of cloudy HRV pixels that are assigned as clear by the MPEF cloud mask, we found an amount of $10 \%$ over the Upper Rhine Valley. This frequency demonstrates the number of small-scale cumulus clouds missed by the MPEF cloud mask (false positive) divided by the number of all clouds (true positive + false positive).

The HRV channel is not used by the EUMETSAT cloud detection algorithm. To demonstrate that its broad spectral response is still suitable for an accurate threshold-based

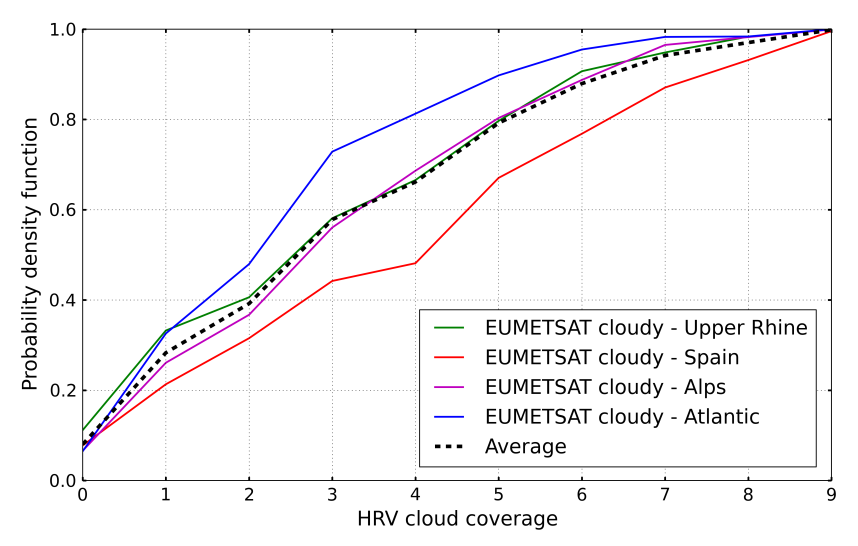

Fig. 7. Cloud detection frequency of the EUMETSAT cloud mask versus the number of cloudy HRV pixels detected by the HRV cloud mask for a corresponding $3 \times 3 \mathrm{HRV}$ pixel block. The solid colored lines mark the average obtained for all HRV pixel blocks for the four regions between 1 June 2011 and 16 August 2011. The average over all regions is indicated by the dotted line.

cloud detection, we have compared the results of our algorithm applied to the HRV channel to those obtained with the narrowband channels at 0.6 and $0.8 \mu \mathrm{m}$ wavelength and at LRES spatial resolution (Fig. 9). For this purpose, the HRV channel is simulated as a linear combination of the 0.6 and $0.8 \mu \mathrm{m}$ reflectances as proposed by Cros et al. (2006) and using the regression coefficients reported by Deneke and Roebeling (2010). The accuracy of the cloud mask applied to the simulated HRV channel lies between those achieved with the 0.6 and $0.8 \mu \mathrm{m}$ channels. Over ocean, differences are small, and the best accuracy is found for the $0.8 \mu \mathrm{m}$ channel, as it is slightly darker than the other channels. Over land, best results are obtained with the $0.6 \mu \mathrm{m}$ channel, but the accuracy of the simulated HRV signal is only slightly lower. Over vegetated surfaces, the $0.8 \mu \mathrm{m}$ exhibits a significantly lower skill, while the relatively bright surface over Spain causes an overall degradation of detection accuracy.

Table 2 summarizes the results of our threshold algorithm and lists the final deviations versus the EUMETSAT cloud 
(a)

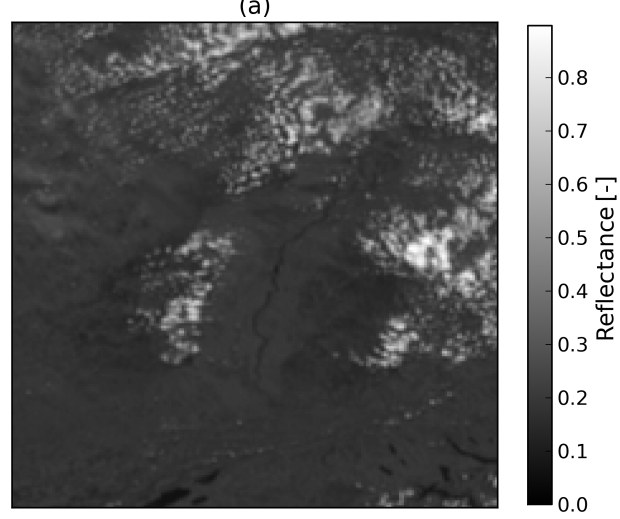

(c)

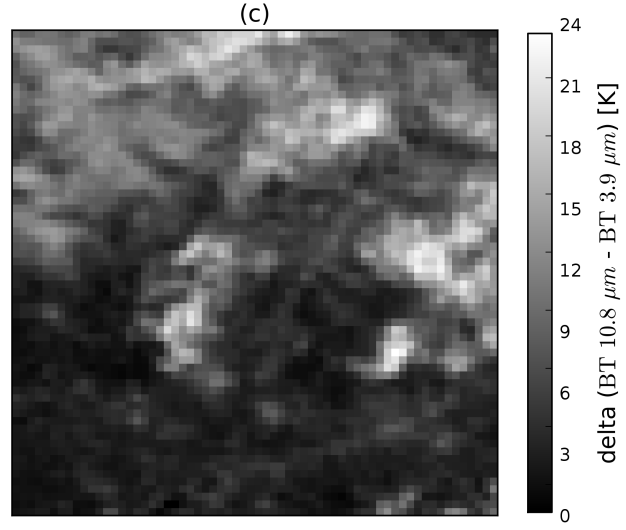

(b)

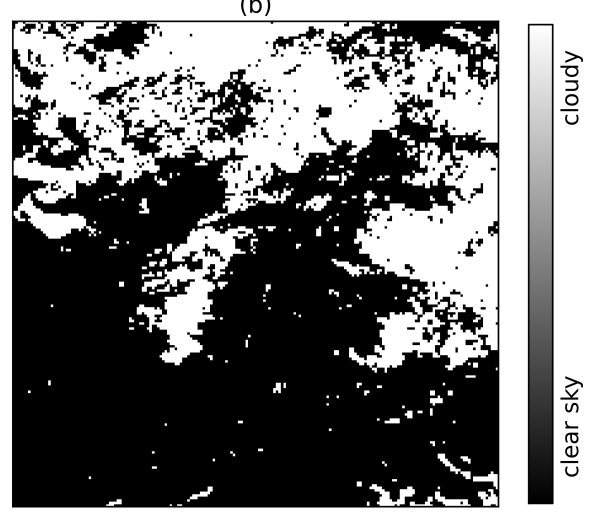

(d)

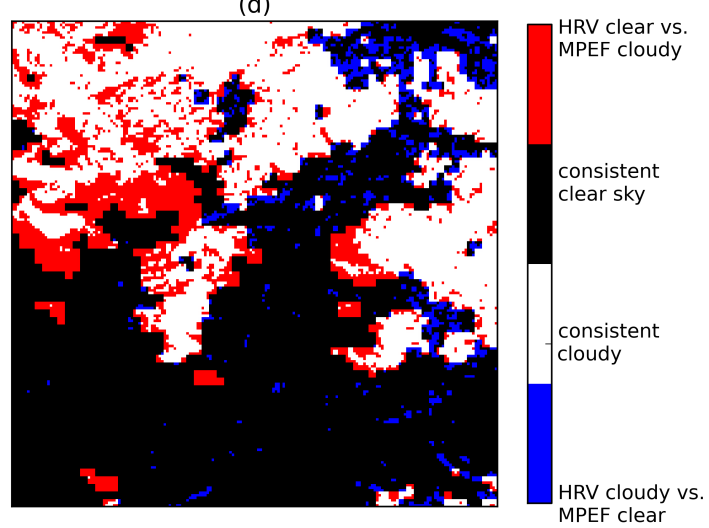

Fig. 8. (a) SEVIRI HRV reflectance over the Upper Rhine Valley on 5 July 2011, 12:00 UTC and (b) HRV cloud mask (white: cloudy, black: clear-sky). (c) Corresponding brightness temperature difference BT $10.8 \mu \mathrm{m}-\mathrm{BT} 3.9 \mu \mathrm{m}$ and (d) difference between MPEF and HRV cloud mask (white: cloudy, black: clear-sky, red: HRV clear-sky versus MPEF cloudy and blue: HRV cloudy versus MPEF clear-sky).

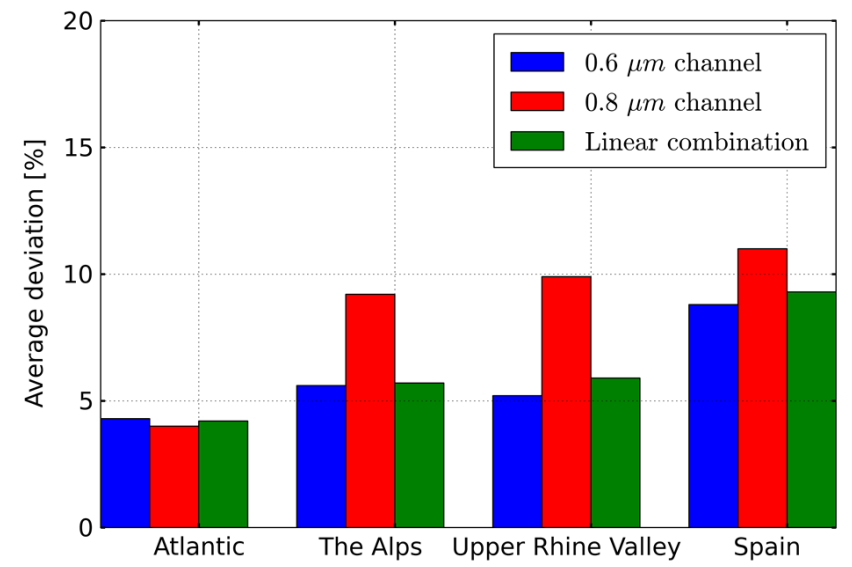

Fig. 9. Average deviation of the EUMETSAT cloud mask and our threshold-based cloud mask algorithm at low resolution and applied to the 0.6 and $0.8 \mu \mathrm{m}$ channels and a linear combination of both channels to simulate the HRV signal (see text for details). Results have been calculated for three periods of 16 days starting on 1 June, 1 July and 1 August 2011. mask. Based on the EUMETSAT cloud mask, all regions but Spain have a high average cloud cover ranging from 75 to $85 \%$. The Atlantic (1) is a region with frequent passages of frontal systems. The Alps (2) and Upper Rhine Valley (3) are characterized by orographically induced convection. One should recognize that the 12:00 UTC time slot is used for this study, which implies a high level of solar irradiance and thus a well-mixed convective boundary layer (Driedonks, 1982). In contrast, the cloud coverage over northern Spain is relatively low. The large observed differences in average cloud cover for the four regions illustrate the importance of choosing a threshold selection scheme that is insensitive to the relative occurrence frequencies of both classes (see Sect. 3.2).

The high standard deviation of the clear-sky reflectance over the Alps and Spain underlines the high spatial variability of the surface over these regions. This finding correlates with a strong reduction of the thresholds $t_{\mathrm{abs}}$ and $t_{\text {rel }}$. Here, $t_{\mathrm{abs}}$ is the absolute threshold determined without using the clear-sky composite information, while $t_{\text {rel }}$ is the threshold relative to the clear-sky composite. Both the lower threshold $t_{\text {rel }}$ compared to $t_{\mathrm{abs}}$, and the resulting lower deviation $\left(\operatorname{Dev}_{\text {rel }}\right)$ compared to Dev $_{\text {abs }}$ confirm that our choice of using a clear-sky composite improves the separability of clear 

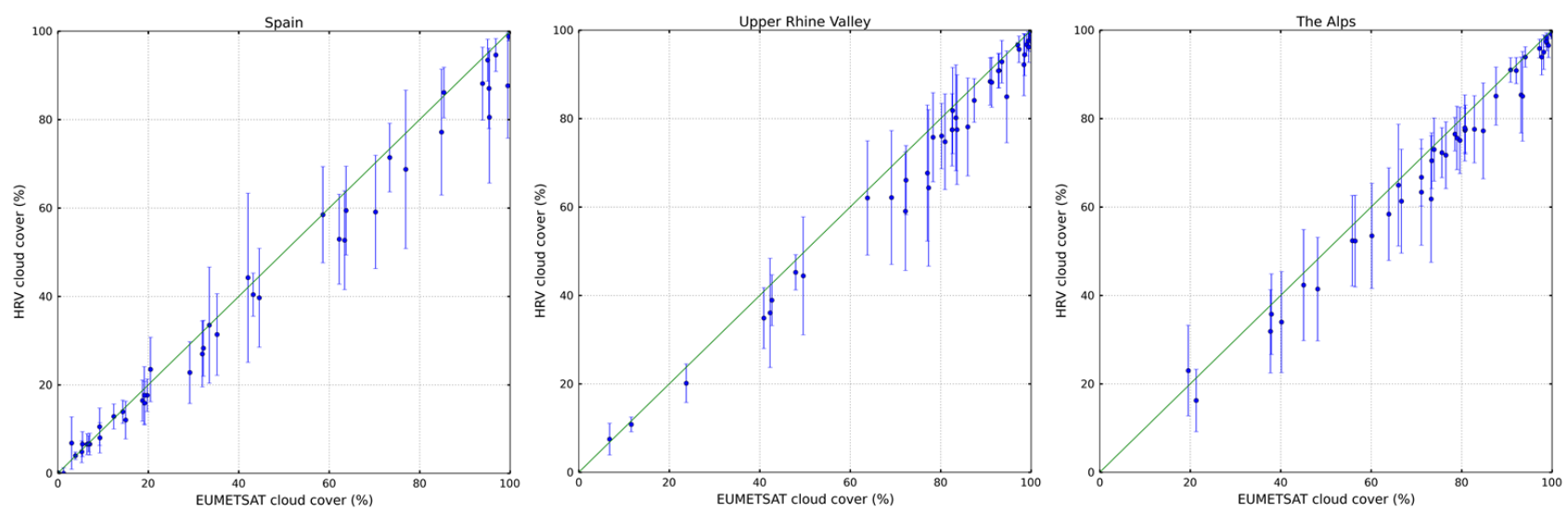

Fig. 10. Comparison of the cloud coverage of the EUMETSAT and HRV cloud masks after thin cloud restoral. The blue dots show the cloud coverage, and the error bars illustrate the fraction of deviating HRV pixel classifications, obtained for each time slot and given in percent. Results have been obtained for three periods of 16 days (starting 1 June, 1 July and 1 August 2011).

and cloudy radiances, and thus results in an overall improved classification accuracy.

Even though the clear-sky variability over the Atlantic region is very low with a standard deviation of only 0.007 , there is still a significant spread of 0.013 between $t_{\mathrm{abs}}$ and $t_{\text {rel }}$. This effect is caused by the initial clear-sky composite, which includes some brighter pixels corresponding to small clouds missed by the EUMETSAT cloud mask. By applying our iteration scheme, these bright pixels are filtered out, as is reflected by a reduced standard deviation of the clear-sky reflectance.

$\operatorname{Dev}_{\mathrm{fi}}$ is the final deviation after applying the thin cloud restoral. The difference between $\operatorname{Dev}_{\text {fi }}$ and $\operatorname{Dev}_{\text {rel }}$ is lowest over the Alps $(1.7 \%)$ and the Upper Rhine Valley $(2.3 \%)$. This is probably due to the high amount of clouds in general, and convective clouds in particular over these regions, which limit the applicability of the thin cloud restoral. On the other hand, a strong impact of the thin cloud restoral on the deviation of the final HRV cloud mask is found for the Atlantic and Spain. Sample scenes such as Fig. 8 indicate a relatively high amount of thin clouds over these regions.

To identify the overall effect of including the HRV channel for cloud masking, Fig. 10 compares the cloud coverages obtained from the EUMETSAT and HRV cloud masks for the three land regions. In the majority of cases, the average HRV cloud coverage lies below the cloud coverage of the EUMETSAT cloud mask. This systematic difference is composed of two effects: first, thin cirrus clouds that are missed by the HRV cloud mask but at least partly corrected for by the thin cloud restoral; second, SEVIRI LRES pixels that are counted as completely cloud-filled by the EUMETSAT cloud mask, but are identified as broken by the HRV cloud mask. While it is impossible to separate both effects without independent reference data, the latter seems to dominate.

The blue error bars indicate the fraction of deviating classifications between both cloud masks found for each time slot.
Generally, the fraction rises with increasing cloud amount, until it reaches a value of about $80 \%$ where it starts to fall again. Thus, partly cloudy conditions cause the highest deviations. This result is expected, because the HRV cloud mask can gain additional information about partly cloudfilled pixels and cloud edges compared due to its higher spatial resolution.

\section{Summary and conclusions}

In this study we have presented and evaluated a thresholdbased HRV cloud mask that is based on the EUMETSAT cloud mask and extends it to a 3-fold higher spatial resolution while maintaining consistency with its results. The optimal threshold to differentiate between clear-sky and cloudy radiances is chosen by maximizing the Matthews correlation coefficient (MCC), a quality measure for binary classifications that is not influenced by the ratio of cloudy to clear pixels, to ensure the best agreement of our cloud mask with the EUMETSAT cloud mask. Clear-sky anomaly maps are used to account for regions with high variability in surface reflectance. As a result, the overlap in the clear and cloudy histograms, and thus the uncertainty in the classification, is significantly reduced. An iterative approach is chosen to include the HRV cloud mask information in the calculation of the clear-sky anomaly maps, with convergence generally achieved after two iterations.

A thin cloud restoral is done to account, for example, for thin cirrus clouds that are not detected by the high-resolution visible channel, in order to ensure that the HRV cloud mask results are consistent with the EUMETSAT cloud mask. Completely clear $3 \times 3 \mathrm{HRV}$ pixel blocks are redefined as cloudy if the corresponding LRES pixel is reported as cloudy in the EUMETSAT cloud mask. Some remaining artifacts after this cloud restoral are found, which are explained and illustrated in Fig. 8. These redefined pixel blocks are flagged 
in a different way than the cloudy pixels that are introduced by the HRV cloud mask. The thin cloud restoral causes some biases, which have been mentioned already. Our future plan is to use the NWCSAF cloud type description to improve the detection of thin cirrus clouds in the HRV cloud mask (Dybbroe et al., 2005). The HRV cloud mask aims to resolve sub-pixel small-scale clouds that are missed by the MPEF cloud mask. In our example, we found an amount of $10 \%$ of these small-scale cumulus clouds over the Upper Rhine Valley. This study shows that the HRV channel offers important sub-pixel information for the remaining low-resolution channels.

On average, $10 \%$ of all $3 \times 3$ clear-sky HRV pixel blocks are missed by our threshold test and restored to cloudy pixels, which occurs mainly for thin cirrus clouds. Our results indicate that the HRV cloud mask performs very reliably in cloudy conditions. The frequency of cloudy LRES pixels that are found to be broken in our data set is $16 \%$. The highest frequency with $24.3 \%$ occurs over the Alps and the lowest fraction over the Atlantic (4.6\%). The amount of broken pixels reaches $15.5 \%$ over Spain and $19.4 \%$ over the Upper Rhine Valley. The high values over the Upper Rhine Valley and the Alps are expected and underline the frequent occurrence of small-scale convective cumuli clouds over these regions. Deviations between the EUMETSAT cloud mask and the HRV cloud mask after thin cloud restoral occur for $5.8 \%$ of the HRV pixels. This deviation results from an overestimate of the cloud fraction due to partially cloudy HRV pixel blocks, which are reported as completely cloudy by the EUMETSAT cloud mask.

This HRV-based cloud mask is part of our wider effort to extend the cloud physical properties retrieval (Roebeling et al., 2006; Roebeling et al., 2008) to the high spatial resolution of the HRV channel, including an estimate of cloud optical thickness (Carbajal Henken et al., 2011) and other cloud properties. It offers also the possibility of applying the cloud mask as a tool to study the geometric size of convective clouds including their temporal evolution in the future. Similar approaches will be essential to utilize the data from future satellite missions optimally, such as Meteosat Third Generation, whose imager has different spatial resolutions for the solar and infrared channels. For a disk-wide application of the HRV cloud mask, more regions with a higher amount of cirrus clouds or high aerosol loads should be considered.

To validate the HRV cloud mask, we have used the MPEF cloud mask as a reference and pointed out the consistency between both masks, but they have different limitations. The cloud retrieval evaluation workshop (CREW) introduces some studies that will be used as independent validation data for the HRV cloud mask in further research (Roebeling et al., 2012). However, more validation has to be done to quantify the accuracy of the HRV cloud mask and the improvements of using the HRV channel for cloud detection schemes. Ideally, the comparison should include MODIS and CloudSat data as has already been done with the MPEF cloud mask (Ricciardelli et al., 2008).

Acknowledgements. The authors thank A. Macke and A. Hünerbein of the Leibniz Institute for Tropospheric Research (TROPOS), who provided many helpful comments and suggestions on previous versions of this paper. The Meteosat- 9 data used in this study were provided by EUMETSAT. We thank the two anonymous reviewers for improving this paper.

Edited by: A. Kokhanovsky

\section{References}

Carbajal Henken, C., Schmeits, M. J., Deneke, H. M., and Roebeling, R. A.: Using MSG-SEVIRI cloud physical properties and weather radar observations for the detection of $\mathrm{Cb} / \mathrm{TCu}$ clouds, J. Appl. Meteorol. Clim., 50, 1587-1600, 2011.

Cros, S., Albuisson, M., and Wald, L.: Simulating Meteosat-7 broadband radiances using two visible channels of Meteosat-8, Sol. Energy, 80, 361-367, 2006.

Deneke, H. M. and Roebeling, R. A.: Downscaling of METEOSAT SEVIRI 0.6 and $0.8 \mu \mathrm{m}$ channel radiances utilizing the highresolution visible channel, Atmos. Chem. Phys., 10, 9761-9772, doi:10.5194/acp-10-9761-2010, 2010.

Derrien, M., Gléau, H. L., and Raoul, M.-P.: The use of the high resolution visible in SAFNWC/MSG cloud mask, in: Proceedings of the 2010 EUMETSAT Meteorological Satellite Conference, 20-24 September 2010, Córdoba, Spain, 57 pp., 2010a.

Derrien, M., Gléau, H. L., and Fernandez, P.: Algorithm theoretical basis document for Cloud products (CMaPGE01 v3.0, CT-PGE02 v2.0 \& CTTH-PGE03 v2.1), ftp://ftp.eumetsat.int/pub/MET/out/watts/SAFMeteoF/ SAF-NWC-CDOP-MFL-SCI-ATBD-01_v3.1d.pdf (last access: 5 June 2013), 2010b.

Driedonks, A. G. M.: Sensitivity analysis of the equations for a convective mixed layer, Bound.-Lay. Meteorol., 22, 475-480, 1982.

Dybbroe, A., Karlsson, K. G., and Thoss, A.: NWCSAF AVHRR cloud detection and analysis using dynamic thresholds and radiative transfer modeling, Part I: Algorithm description, J. Appl. Meteorol., 44, 39-54, 2005.

EUMETSAT: Cloud Detection for MSG - Algorithm Theoretical Basis Document, http://www.eumetsat.int/groups/ ops/documents/document/PDF_MET_ATBD_CLOUD_

DETECTIO N.pdf (last access: 14 August 2012), 2007.

Forster, P., Ramaswamy, V., Artaxo, P., Berntsen, T., Betts, R., Fahey, D. W., Haywood, J., Lean, J., Lowe, D. C., Myhre, G., Nganga, J., Prinn, R., Raga, G., Schulz, M. and Van Dorland, R.: Changes in Atmospheric Constituents and in Radiative Forcing, in: Climate Change 2007: The Physical Science Basis, Contribution of Working Group I to the Fourth Assessment Report of the Intergovernmental Panel on Climate Change, edited by: Solomon, S., Qin, D., Manning, M., Chen, Z., Marquis, M., Averyt, K. B., Tignor, M. and Miller, H. L., Cambridge University Press, Cambridge, UK and New York, NY, USA, 129-234, 2007. 
Frey, R., Ackerman, S. A., Liu, Y., Strabala, K. I., Zhang, H., Key, J., and Wang, X.: Cloud detection with MODIS: Part I. Improvements in the MODIS Cloud Mask for Collection 5, J. Atmos. Ocean. Tech., 25, 1057-1072, 2008.

Ipe, A., Clerbaux, N., Bertrand, C., Dewitte, S., and Gonzalez, L.: Pixel-scale composite top-of-the-atmosphere clear-sky reflectances for Meteosat-7 visible data, J. Geophys. Res., 108, 4612, doi:10.1029/2002JD002771, 2003.

Klüser, L., Rosenfeld, D., Macke, A., and Holzer-Popp, T.: Observations of shallow convective clouds generated by solar heating of dark smoke plumes, Atmos. Chem. Phys., 8, 2833-2840, doi:10.5194/acp-8-2833-2008, 2008.

Lacis, A. A. and Hansen, J. E.: A parameterization for the absorption of solar radiation in the Earth's atmosphere, J. Atmos. Sci., 31, 118-133, 1974.

Lensky, I. M. and Rosenfeld, D.: Clouds-Aerosols-Precipitation Satellite Analysis Tool (CAPSAT), Atmos. Chem. Phys., 8, 6739-6753, doi:10.5194/acp-8-6739-2008, 2008.

Liou, K. N.: An Introduction to Atmospheric Radiation, in: International Geophysics Series 25, Academic Press, 392 pp., 1980.

Matthews, B. W.: Comparison of the predicted and observed secondary structure of T4 phage lysozyme, Biochim. Biophys. Acta, 405, 442-451, 1975.

Minnis, P. and Harrison, E. F.: Diurnal variability of regional cloud and clear-sky radiative parameters derived from goes data, Part iii: November 1978 radiative parameters, J. Clim. Appl. Meteorol., 23, 993-1011, 1984.

Ricciardelli, E., Romano, F., and Cuomo, V.: Physical, statistical and temporal analysis approaches for cloud identification using MSG-SEVIRI data, in: Proceedings of the 2008 EUMETSAT Meteorological Satellite Conference, 8-12 September 2008, Darmstadt, Germany, 2008.
Roebeling, R. A. and van Meijgaard, E.: Evaluation of the daylight cycle of model predicted cloud amount and condensed water path over Europe with observations from MSG-SEVIRI, J. Climate, 22, 1749-1766, 2009.

Roebeling, R. A., Feijt, A. J., and Stammes, P.: Cloud property retrievals for climate monitoring: implications of differences between SEVIRI on METEOSAT-8 and AVHRR on NOAA-17, J. Geophys. Res., 111, 206-222, 2006.

Roebeling, R. A., Deneke, H., and Feijt, A. J.: Validation of cloud liquid water path retrievals from SEVIRI using one year of CloudNET observations, J. Appl. Meteorol. Clim., 47, 206-222, 2008.

Roebeling, R., Baum, B., Bennartz, R., Hamann, U., Heidinger, A., Thoss, A., and Walther, A.: Outcome of the third cloud retrieval evaluation workshop, in: Proceedings of the 2012 Radiation Processes in the Atmosphere and Ocean (IRS2012), 6-10 August 2012, Berlin, Germany, 416-419, doi:10.1063/1.4804795, 2012.

Rossow, W. B., Garder, L. C., and Lacis, A. A.: Global, seasonal cloud variations from satellite radiance measurements, Part I: Sensitivity of analysis, J. Climate, 2, 419-458, 1989.

Saunders, R. W. and Kriebel, K. T.: An improved method for detecting clear sky radiances from AVHRR data, Int. J. Remote Sens. 9, 123-150, 1988.

Schmetz, J. P., Pili, P., Tjemkes, S., Just, D., Kerkmann, J., Rota, S., and Ratier, A.: An introduction to Meteosat Second Generation (MSG), B. Am. Meteorol. Soc., 83, 977-992, 2002.

Yang, Y., Girolamo, L., and Mazzoni, D.: Selection of the automated thresholding algorithm for the Multi-angle Imaging SpectroRadiometer Radiometric Camera by - Camera Cloud Mask over land, Remote Sens. Environ., 107, 159-171, 2007. 